\title{
Impact of Elliptical Cross-Section on Some Electrical Properties of Gate-All-Around MOSFETs
}

\author{
Shankaranand Jha, Ashok Kumar and Subindu Kumar
}

\begin{abstract}
Silicon (Si) Gate-All-Around (GAA) MOSFETs offers full electrostatic control over the gate which makes them promising candidates for the next generation complimentary metal-oxide-semiconductor field-effect transistors (CMOS) devices. Due to variations in the growth condition, the cross-section of GAA MOSFETs is often elliptical instead of being perfectly circular. This elliptical cross section changes the effective diameter of the silicon core which in turn affects the electrical properties of the device. In this paper we analyze the impact of elliptical cross-section of multi-channel Si GAA MOSFET on some electrical properties such as threshold voltage, drain current, and so on. The dependence of such properties on doping concentration, thickness of the oxide (insulator), channel dimension and number of conducting channels are also presented.
\end{abstract}

Keywords--- Aspect Ratio, Effective Diameter, MOSFET, Multi-Channel

\section{INTRODUCTION}

$\mathrm{T}$ HE near-ideal characteristics of Gate-All-Around (GAA) MOSFET makes it a promising candidate for the nextgeneration nanoscaled circuits. The symmetry of GAA MOSFET has enabled the research community to go in for various approximations in the derivation of the characteristics of the device which would otherwise have been very complex. GAA MOSFETs do not have corner effects [1] due to the circular cross-section and cylindrical body. Furthermore, by solving the Poission/drift-diffusion equation in cylindrical coordinates, the electrical characteristics of GAA devices can be modeled analytically $[2,3]$. Recently CMOS compatible multichannel (MC) GAA MOSFETs having diameters $\leq 5 \mathrm{~nm}$ has been demonstrated with excellent performance [4].

Due to limitations in the fabrication process, the cross section of GAA devices is not circular. The fabricated structures may have elliptical cross section with different major and minor axis lengths [5-7]. The geometric aspect ratio

Shankaranand Jha, Junior Research Fellow, Department of Electronics Engineering, Indian School of Mines, Dhanbad - 826004, India. E-mail: shankaranand_ism@yahoo.co.in

Ashok Kumar, Assistant Professor, Department of Electronics and Telecommunication Engineering, Dr. D.Y. Patil School of Engineering, Pune, India.E-mail:ashok1987.in@gmail.com

Subindu Kumar, Assistant Professor, Department of Electronics Engineering, Indian School of Mines, Dhanbad - 826004, India. E-mail: kumar.s.ei@ismdhanbad.ac.in
(AR) of the elliptical GAA MOSFET is defined as the ratio of the length of the major axis $(a)$ to that of the minor axis $(b)$. As a special case, when $a$ and $b$ are equal, the structure is perfectly circular. The ideally circular cross section of GAA MOSFET may not give the optimized circuit performance. The effect of elliptical cross-section on the electrical characteristics have not been widely discussed or analyzed. In this paper we emphasize on the electrical characteristics of MC elliptical GAA MOSFETs for a wide range of diameters.

\section{LITERATURE SURVEY}

There are several research papers in the frontier of modern electronics which deals with the fabrication, theoretical modeling, and analysis of perfectly circular GAA MOSFETs [8-10]. The cross-section of fabricated GAA devices, which take part in real time circuit operation, may be elliptical. There are few reports which have emphasized on the modeling and analysis of such elliptical GAA devices. The pioneer works on elliptical GAA MOSFETs include the studies of $\mathrm{Li}$ and Hwang [11] on the effects of geometry AR on elliptical GAA transistor using 3D coupled device-and-circuit simulations.

The universality of short-channel effects in undoped-body silicon nanowire MOSFETs having elliptical cross section was proposed by Bangsaruntip et al. [12]. They have shown that the short-channel effects of undoped-body GAA MOSFETs can be improved by shrinking the body dimensions. Zhang et al. [13] proposed the concept of effective radius to convert elliptical GAA MOSFETs into equivalent circular GAA

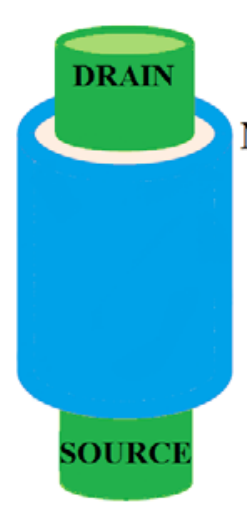

(a)
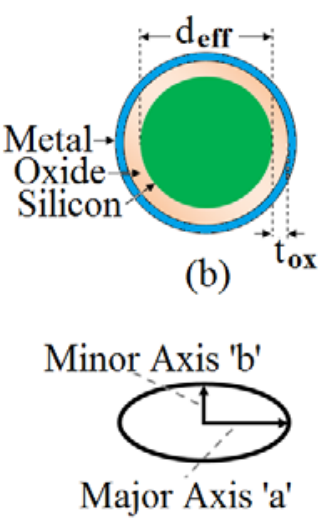

(c)
Figure 1: (a) Structure of GAA MOSFET, (b) Cross sectional view and (c) Schematic of an elliptical GAA with the major axis length, ' $a$ ' and minor axis ' $b$ '. 
MOSFETs for studying short-channel effects. They found that the elliptical GAA MOSFETs that have the same effective radius exhibit the same short channel effects and $\mathrm{I}_{\mathrm{ON}} / \mathrm{I}_{\mathrm{OFF}}$ ratio.

\section{THEORETICAL DETAILS}

Figure 1(a) represents the structure of a GAA MOSFET. The cross section is shown in Figure 1(b). Figure 1(c) represents the schematic of an elliptical GAA with the major axis length, ' $a$ ' and minor axis ' $b$ '. The aspect ratio is defined as $a / b$. The overall threshold voltage, $V_{t h}$, of the GAA MOSFET is given by $V_{t h}=V_{t h 0}+\Delta V_{t}+\Delta V_{Q M}[14],[15]$. $V_{t h 0}$ is the threshold voltage that is similar to that of the bulk MOSFETs [16] and $\Delta V_{t}$ is the additional component of the threshold voltage due to the special geometry of GAA MOSFETs given by [14]

$$
\begin{aligned}
& V_{t h 0}=\Delta \varphi+2 \varphi_{f}+\frac{Q_{d e p}}{C_{O X}}-\frac{1}{\beta} \ln \left(\frac{2 \beta q N_{a} \varepsilon_{S i}}{C_{O X}^{2}}\right) \\
& \Delta V_{t}=-\left(\frac{1}{\beta}\right) \ln \left(\frac{2 C_{o x}}{\beta q N_{a} d_{e f f}}\right)\left[1-\exp \left(-\frac{q^{2} N_{a} d_{e f f}^{2}}{16 \varepsilon_{s i} k T}\right)\right]
\end{aligned}
$$

where

$\Delta \phi \quad$ work function difference between the gate material and silicon body;

$C_{O X} \quad 2 \varepsilon_{O X}\left[d_{e f f} \ln \left(1+2 t_{O X} / d_{\text {eff }}\right)\right] . \quad$ Effective oxide capacitance per unit gate area;

$t_{O X} \quad$ thickness of the gate oxide;

$d_{\text {eff }} \quad$ effective diameter of the elliptical silicon body [13];

$C_{\text {total }}$ total oxide capacitance;

$\phi_{f} \quad$ Fermi potential;

$Q_{\text {dep }} \quad q N_{a} d_{e f f} / 4$. Depletion charge density per unit gate area;

$N_{a} \quad$ impurity concentration in the silicon body;

$(1 / \beta)=k T / q$. Thermal voltage.

$\Delta V_{Q M}$ is another additional component which takes into account the changes in $V_{\text {th }}$ due to the nanometric diameter of GAA devices. Other variables, such as $n_{i}, q, k, T, \varepsilon_{O X}$, and $\varepsilon_{S \mathrm{~S}}$, have their usual physical meanings. The saturated drain current, $I_{D S a t}$ of a coaxially gated n- channel GAA MOSFET can be expressed as [14]:

$$
\begin{aligned}
& I_{D S a t} \approx \frac{\mu\left(\pi d_{e f f}\right)}{L} C_{O X}\left[\frac{1}{2}\left(V_{g s}-V_{t h}\right)^{2}-\frac{1}{\beta^{2}} e^{\beta\left(V_{g s}-V_{t h}-V_{d s}\right)}\right] \\
& \quad \approx \frac{\pi d_{e f f} \mu}{2 L} C_{O X}\left(V_{g s}-V_{t h}\right)^{2}
\end{aligned}
$$

where

$\mu$ mobility of the charge carriers under consideration;

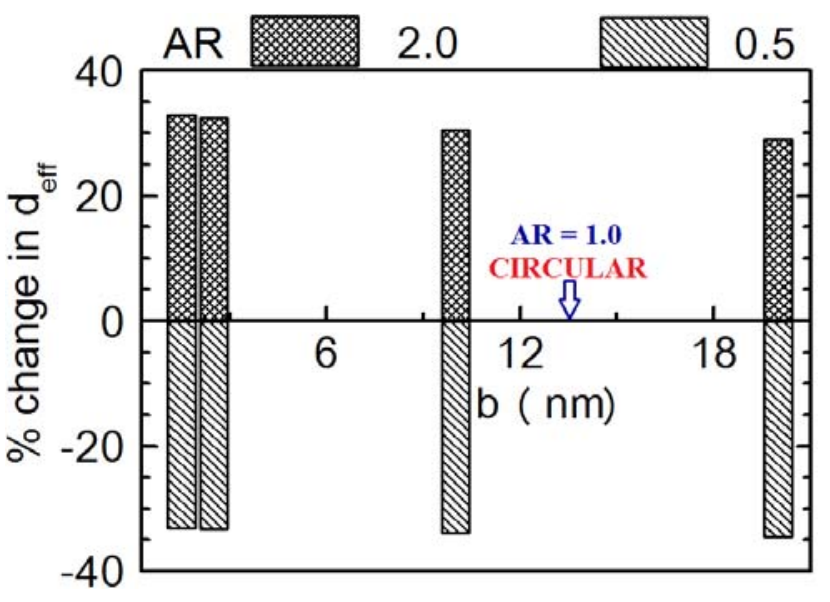

Figure 2: Percentage change in effective diameter with minor axis ' $b$ ' corresponding to $\mathrm{AR}=2.0$ and 0.5 .

\section{$L \quad$ channel length of the GAA structure;}

For our computations we have considered a $1 \mu \mathrm{m}$ long nchannel GAA MOSFET with $\mathrm{SiO}_{2}$ as the gate dielectric. It is worth mentioning here that in this work, we have considered long channel devices, which are still of technological importance, to eliminate short channel effects so that the contribution of GAA structure is highlighted. Since the geometry AR of elliptical GAA structure is seldom larger than 2.0 [13] and in order to keep our computation within presentable limits, we have varied the AR from 0.5 to 2.0. This range of AR is obtained by keeping ' $b$ ' fixed and varying ' $a$ ' for a particular set of computation. As a special case, $\mathrm{AR}=1.0$ gives us a circular cross section.

\section{RESULTS AND DisCUSSION}

The results of our computations for $\mathrm{n}$-channel devices are shown in Figures 2-9. The percentage change in effective diameter for various minor axis, corresponding to $\mathrm{AR}=2.0$ and 0.5 is depicted in Figure 2. $0 \%$ corresponds to a perfectly circular cross-section having AR $=1.0$. For AR $>1.0$, there is an increase in the effective diameter which is shown in the positive axis of Figure 2. For AR $<1.0$, the results of our computations are reversed. This is in consistence with the results reported by Zhang et al [13].This variation in the effective diameter in turn affects the threshold voltage and drain current of an elliptical GAA device. Figure 3 represents the variation of the threshold voltage of nMOS GAA with channel doping concentration for AR ranging from 0.5 to 2.0 corresponding to $b=1.5$ and $20 \mathrm{~nm}$. A change in the AR results in the change of the effective diameter which in turn changes the threshold voltage of the device. We observe that for both $\mathrm{b}=1.5$ and $20 \mathrm{~nm}, V_{t h}$ corresponding to a particular AR remains constant for lightly and moderately doped channel. For highly doped channel, $V_{t h}$ shoots up for all ARs. The $V_{t h}$ shooting is more pronounced for larger elliptical cross sections. The impact of doping concentration on the threshold voltage of perfectly circular GAA was reported in [14]. We obtained a similar nature as illustrated in Figure 3, where we 


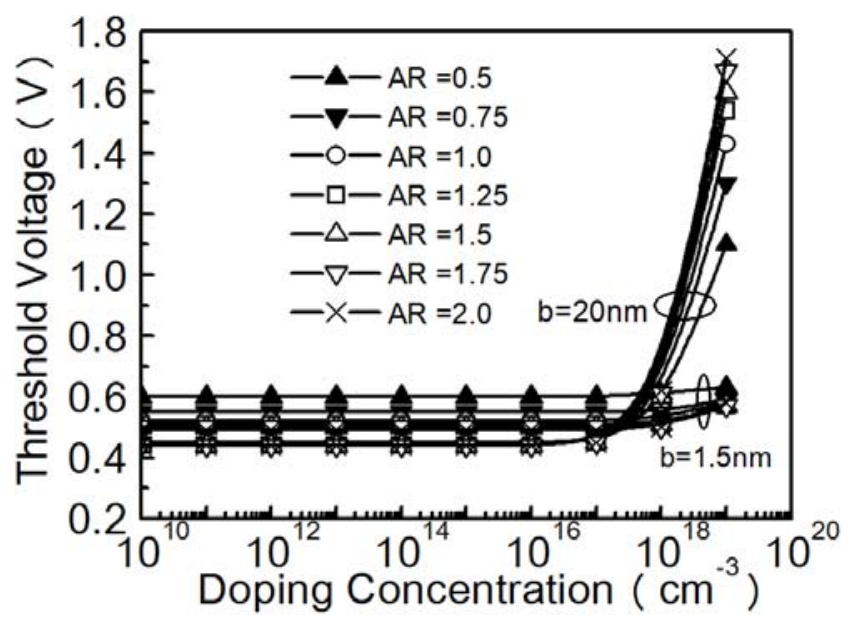

Figure 3: Variation of threshold voltage with channel doping concentration for different ARs corresponding to $b=1.5$ and $20 \mathrm{~nm}$.

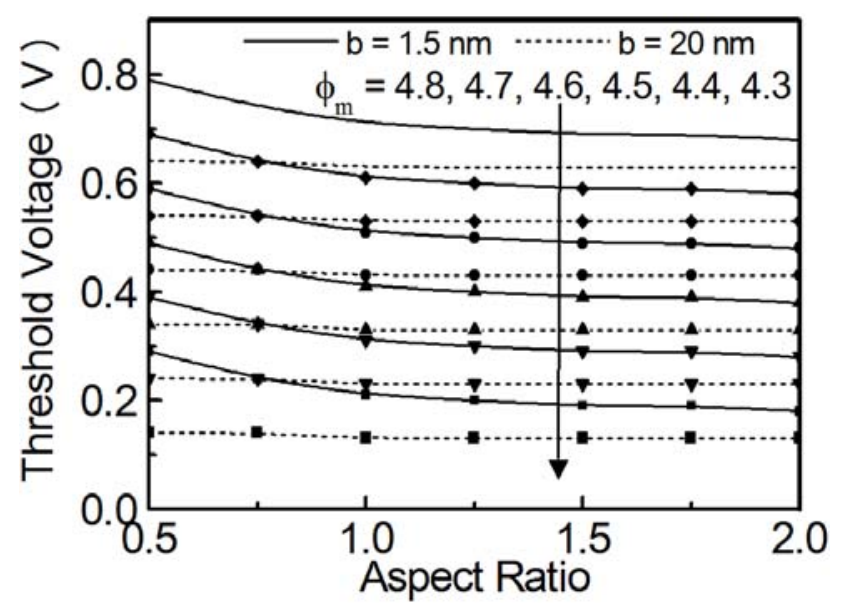

Figure 4: Variation of the threshold voltage with AR for different metal work functions corresponding to $b=1.5$ and $20 \mathrm{~nm}$.

have considered a number of device ARs including a perfectly circular GAA having $\mathrm{AR}=1.0$.

Figure 4 depicts the variation of $V_{t h}$ with AR for different metal work functions, $\phi_{m}$, corresponding to $\mathrm{b}=1.5$ and 20 $\mathrm{nm}$. As expected, the $V_{t h}$ corresponding to a lower value of $\phi_{m}$ for a particular AR is the least. For larger cross sections, there is a monotonic decrease in $V_{t h}$ with AR. It could be inferred that on one hand decreasing $\phi_{m}$ reduces the value of $V_{t h}$ and on the other hand, for larger GAA cross section, $V_{t h}$ increases as we decrease the AR. Selecting a proper value of $V_{t h}$ is a tradeoff between (1) the metal work function and (2) the geometry of the GAA device.

Figure 5 shows the current driving capability of elliptical GAA MOSFET for various ARs corresponding to different minor axis. We have taken into account the saturated drain current which increases monotonically with the AR. We observe that a circular GAA with $\mathrm{AR}=1.0$ is an optimized structure as the current driving capability can be further enhanced by increasing the AR. It is also found that devices

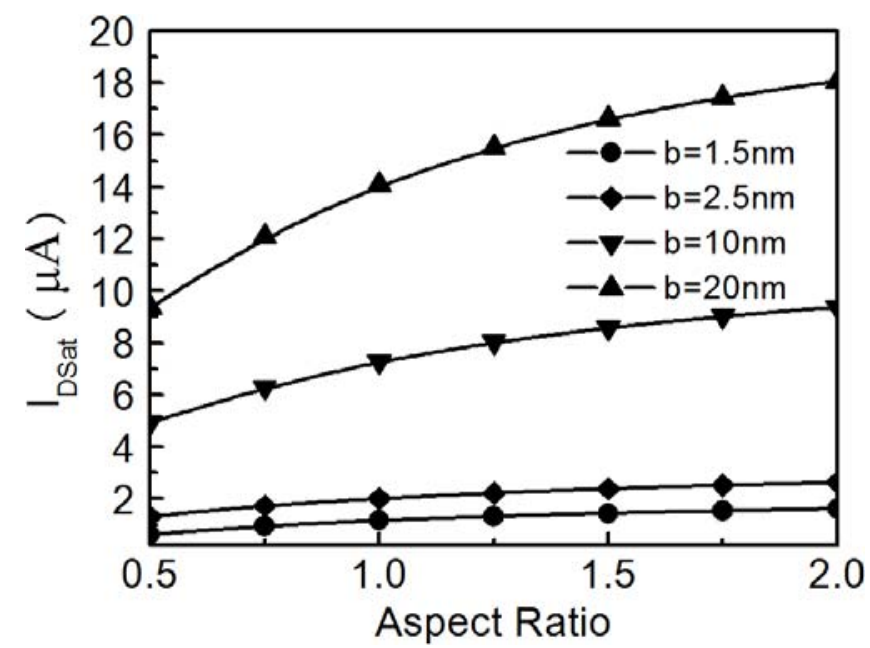

Figure 5: Variation of the saturated drain current with AR for $\mathrm{b}=1.5,2.5,10$ and $20 \mathrm{~nm}$.

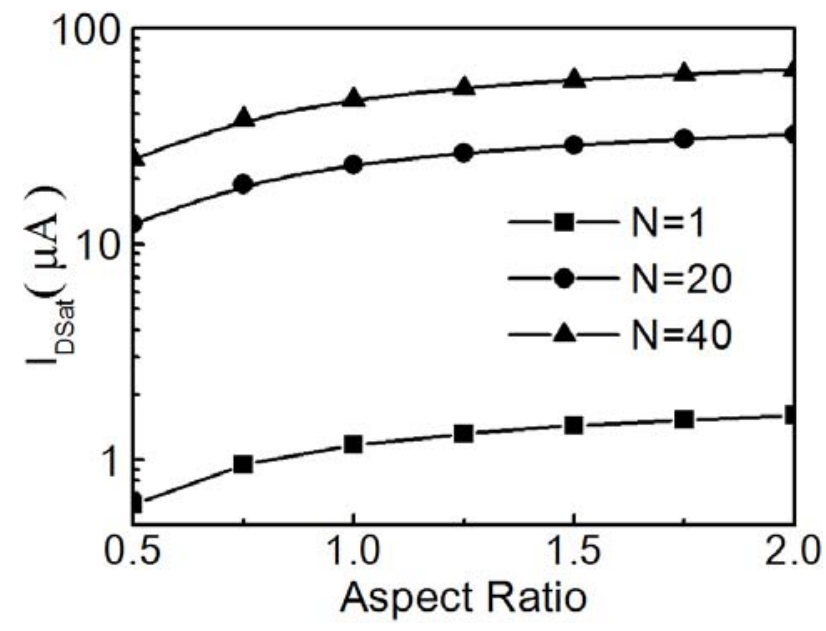

Figure 6: Variation of the saturated drain current with AR for multi-channel GAA MOSFETs. ' $N$ ' denotes the number of channels.

with larger cross section can furnish more current than their smaller counterpart. This is in consistence with (3). In Figure 6 the variation of the saturated drain current with AR is plotted for GAA MOSFETs having number of conducting channel(s) $N=1,20$ and 40. One of the most interesting characteristics of MC GAA devices is that the current driving capability can be increased by increasing the number of channels, keeping the geometrical aspect and the physical dimensions unchanged.

The dependence of the saturated drain current on the oxide thickness for $b=1.5$ and $20 \mathrm{~nm}$ corresponding to various ARs has also been investigated and the results are depicted in Figure 7. The drive current can be increased to a large extent by reducing the oxide thickness. In an effort to increase the drive current on one hand by reducing the oxide thickness beyond $1.5 \mathrm{~nm}$, the gate tunneling current may increase on the other hand. An optimized selection of the oxide thickness is necessary for designing GAA devices. It is also observed that for a fixed oxide thickness, the drive current is more for an AR of 2.0 . 


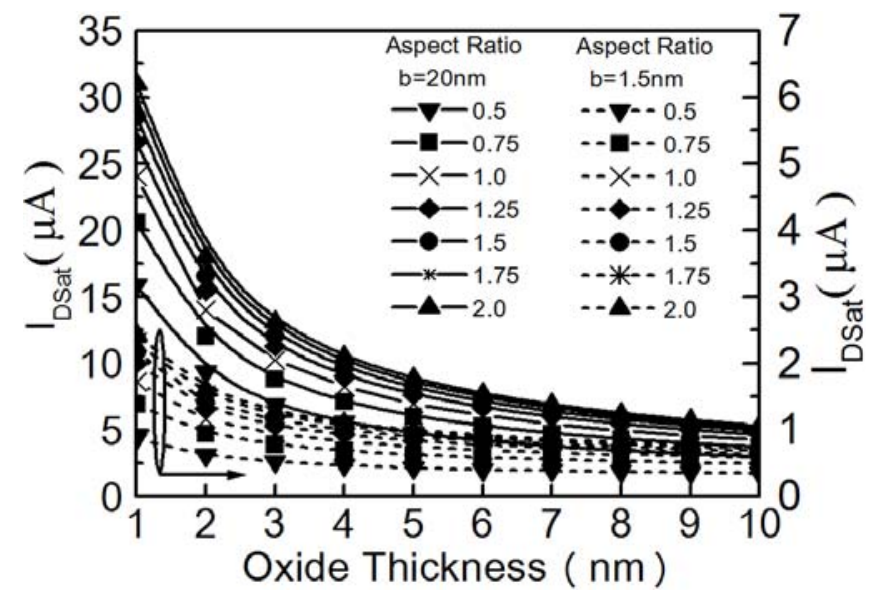

Figure 7: Variation of the saturated drain current with gate oxide thickness for different ARs corresponding to $b=1.5$ and $20 \mathrm{~nm}$.

Inverters form the basic building block of complex digital circuits. The performance of the inverter depends on the structure of the n- and p-MOSFETs. As one of the most important performance parameters in CMOS digital circuits, propagation delay is of concern to designers and users. A simple way of analysing the propagation delay is to replace the device with its On-Resistance, extract the load capacitance and calculate the $R C$ time constant. Several approaches use such strategy for the transient analysis. In this work, we have emphasized on the average On-Resistance, which is an important electrical parameter of coaxially gated MC GAA MOSFET for determining the propagation delay of digital circuits. Figure 8 shows the variation of average OnResistance of n-channel GAA device with AR corresponding to various conducting channels for $b=1.5$ and $20 \mathrm{~nm}$. We observe that the On-Resistance decreases monotonically with AR. Figure 8 also depicts that the On-Resistance of GAA devices can be drastically decreased by increasing the number of conducting channels. These, in turn, affect the transient characteristics of the circuit significantly, as shown in Figure

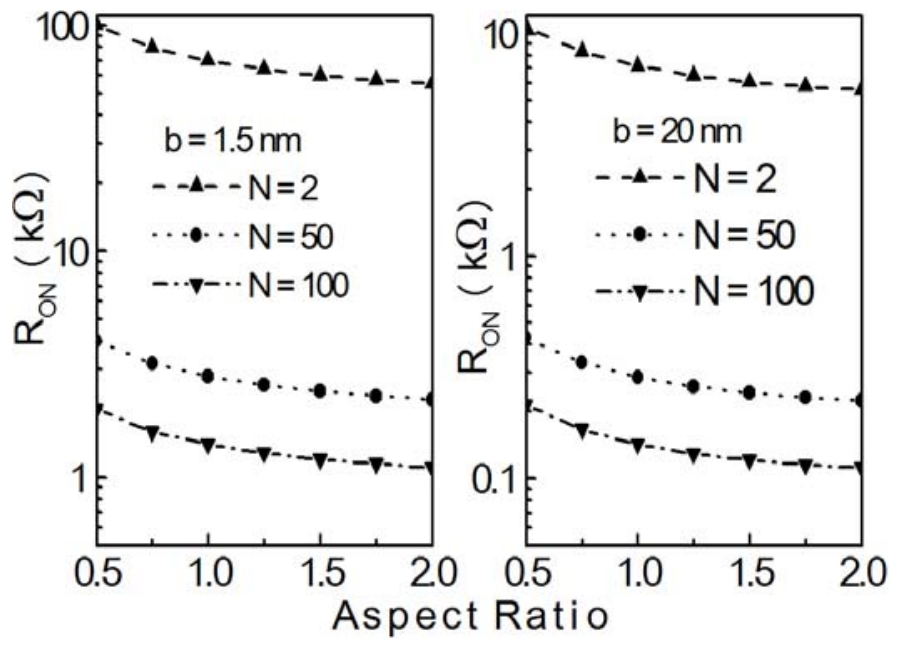

Figure 8: Variation of ON-resistance with AR for number of channels 2,50 and 100 corresponding to $b=1.5$ and $20 \mathrm{~nm}$.

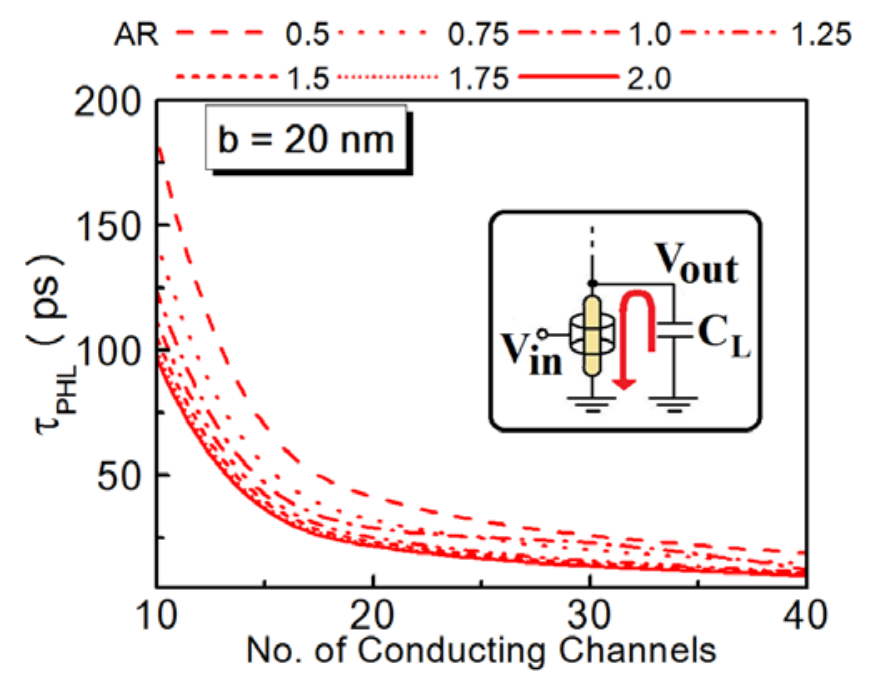

Figure 9: Plot of output fall time of MC n-GAA MOSFET with number of conducting channels corresponding to various

ARs. The inset shows the discharging path from the load

capacitance to ground through n-type GAA device.

9, where the variation of output fall time $\left(\tau_{P H L}\right)$ of n-type $\mathrm{MC}$ elliptical GAA MOSFET is plotted against number of conducting channels corresponding to various ARs. The output fall can be expressed as $\ln (2) \tau_{n}$, where $\tau_{n}\left(=R_{\text {avg, } n} C_{L}\right)$ is the time constant related to the discharging of the n-MOS, as illustrated in the inset of Figure 9. $C_{L}$ is the load capacitance which includes the parasitic output capacitance of the inverter, the interconnect capacitance and the gate capacitance of the next stage circuit. In this work $C_{L}$ has been considered to be $50 \mathrm{fF}$. To validate and compare our approach, we have computed the $\tau_{P H L}$ of GAA MOSFET based circuits with the parameters reported by Buddharaju et al [4], Huang et al [17] and Rustagi et al [8]. The average deviation was found to be less than $5 \%$.

One of the advantages offered by multi-channel GAA MOSFETs is that the drain current can be tuned by solely increasing (decreasing) the number of conducting channels, which in turn modifies $\tau_{P H L}$, and hence the propagation delay of circuits utilizing GAA devices. We observe a drastic reduction in the fall time with increasing number of conducting channels in n-MOS. In this work, we have focused on the fall time of n-type GAA device. However, for calculating the average propagation delay of CMOS circuits, the output rise time needs to be computed. In case of planar CMOS inverters, to compensate for the low value of hole mobility for achieving symmetry in rise and fall times, with fixed gate length $L$, the width $W$ for the p-MOS is kept typically $\sim 2 \times$ higher than that of n-MOS [16]. In case of GAA MOSFETs (similar to the case of FinFETs), the 'diameter' (similar to the 'width' of planar MOSFETs) is fixed and is same for both the n-MOS and the p-MOS devices. Therefore, the low values of hole mobility can be compensated by choosing larger number of channels for p-MOS transistors [4]. To obtain equal rise and fall time of a MC GAA MOSFET based complementary circuit, care has to be taken to increase the number of conducting channels of p- GAA MOSFET 
simultaneously maintaining the ratio $1: 2$. It is also worth mentioning here that output fall time may be brought down to few pico-seconds by increasing the number of conducting channels, as shown in Figure 9. Use of 100 channels per GAA device has also been reported in the literature [4].

\section{CONCLUSION}

To summarize, we have investigated the effect of AR, channel doping concentration, metal work function, number of conducting channels and gate oxide thickness on some electrical parameters of MC elliptical GAA MOSFET. Some techniques are discussed which may be useful for optimized design of GAA devices. Through our computations, we have shown that the transient characteristics of MC GAA devices can be improved by tuning the number of conducting channels. We have also established that GAA MOSFETs having ideally circular cross section do not give the optimized device characteristics.

\section{REFERENCES}

[1] L. Choi, B.H. Hong, Y.C. Jung, K.H. Cho, K.H. Yeo, D-W Kim, G.Y. Jin, K.S. Oh, W-S Lee, S-H Song, J.S. Rieh, D.M. Whang and S.W. Hwang, "Extracting Mobility Degradation and Total Series Resistance of Cylindrical Gate-All-Around Silicon Nanowire Field-Effect Transistors," IEEE Electron Device Lett., Vol. 30, No. 6, Pp. 665-667, June 2009.

[2] B. Iniguez, D. Jimenez, J. Roig, H.A. Hamid, L.F. Marsal and J. Pallares, "Explicit Continuous Model for Long-Channel Undoped Surrounding Gate MOSFETs," IEEE Trans. Electron Devices, Vol. 52, No. 8, Pp. 1868-1873, Aug. 2005.

[3] L. Singh, M. Chandra, and B.P. Tyagi, "Mobility Degradation and Total Series Resistance of Cylindrical Gate-All-Around Silicon Nanowire Field-Effect Transistor,” Intern. Journ. Engg. Mgmt Res., Vol. 2, No.4, Aug 2012.

[4] K.D. Buddharaju, N. Singh, S.C. Rustagi, Selin H.G. Teo, G.Q. Lo, N. Balasubramanian, and D.L. Kwong, "Si-nanowire CMOS inverter logic fabricated using gate-all-around (GAA) devices and top down approach," Solid State Electron., Vol. 52, Pp. 1312-1317, May 2008.

[5] Y. Li, H-M Chou and J-W Lee, "Investigation of electrical characteristics on surrounding-gate and omega-shaped-gate nanowire FinFETs," IEEE Trans. Nanotechnol., Vol. 4, No. 5, Pp. 510-516, Sep. 2005 .

[6] S. Bangsaruntip, G.M. Cohen, A. Majumdar, Y. Zhang, S.U. Engelmann, N. Fuller, L. M. Gignac, S. Mittal, J. S. Newbury, M. Guillorn, T. Barwicz, L. Sekaric, M. M. Frank, and J. W. Sleight, "High performance and highly uniform gate-all-around silicon nanowire MOSFETs with wire size dependent scaling," IEDM Tech Dig., Pp. 297-300, 2009

[7] R.M.Y. Ng, T. Wang, F. Liu, X. Zuo, J. He, and M. Chan, "Vertically stacked silicon nanowire transistors fabricated by inductive plasma etching and stress-limited oxidation," IEEE Electron Device Lett., Vol. 30, No. 5, Pp. 520-522, May 2009.

[8] S.C. Rustagi, N. Singh, W.W. Fang, K.D. Buddharaju, S.R. Omampuliyur, S.H.G. Teo, C. H. Tung, G. Q. Lo, N. Balasubramanian, and D. L. Kwong, "CMOS inverter based on gate-all-around siliconnamowire MOSFETs fabricated using top-down Approach," IEEE Electron Device Lett., Vol. 28, No. 11, Pp. 1021-1024, Nov. 2007.

[9] S.H. Oh, D. Monroe, and J.M. Hergenrother, "Analytical description of short-channel effects in fully-depleted double-gate and cylindrical, surrounding-gate MOSFETs," IEEE Electron Device Lett., Vol. 21, No. 9, Pp. 445-447, Sep. 2000.

[10] Y. Cui, Z. Zhong, D. Wang, W. Wang, and M. Lieber, "High performance silicon nanowire field effect transistors," Nano Lett., Vol. 3, No. 2, Pp. 149-152, Jan. 2003.

[11] Y. Li, and C.-H. Hwang, "The effect of the geometry aspect ratio on the silicon ellipse-shaped surrounding-gate field-effect transistor and circuit," Semicond. Sci. Technol. Vol. 24, No. 9, Pp. 1-8, Aug. 2009.

[12] S. Bangsaruntip, G.M. Cohen, A. Majumdar, and J.W. Sleight, "Universality of short-channel effects in undoped-body silicon nanowire
MOSFETs," IEEE Electron Device Lett,. Vol. 31, No. 9, Pp. 903-905, Sep. 2010.

[13] Lining Zhang, Lin Li, Jin He and Mansun Chan, "Modeling ShortChannel Effect of Elliptical Gate-All-Around MOSFET by Effective Radius," IEEE Electron Device Lett., Vol. 32, No. 9, Pp. 1188-1190, Sep. 2011.

[14] Feng Liu, Jin He, Lining Zhang, Jian Zhang, Jinghua Hu, Chenyue Ma and Mansun Chan, "A charge-based model for long-channel cylindrical surrounding-gate MOSFETs from intrinsic channel to heavily doped body," IEEE Trans. Electron Devices, Vol. 55, No. 8, Pp. 2187-2194, Aug. 2008.

[15] J. B. Roldán, Andrés Godoy, Francisco Gámiz and M. Balaguer, "Modelling the Centroid and the Inversion Charge in Cylindrical Surrounding Gate MOSFETs, Including Quantum Effects," IEEE Trans. Electron Devices, Vol. 55, No.1, Pp. 411-416, Jan. 2008.

[16] N. Arora, "MOSFET Models for VLSI Circuit Simulation Theory and Practice", New York: Springer-Verlag, 1993.

[17] R.G. Huang, D. Tham, D. Wang, and J.R. Heath, "High performance ring oscillators from $10-\mathrm{nm}$ wide silicon nanowire field-effect transistors," Nano Res., Vol. 4, No. 10, Pp. 1005-1012, Dec. 2011.

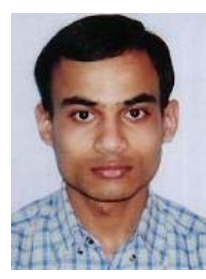

Shankaranand Jha was born in Madhubani, India, in 1986. He received the B.E degree in Electronics \& Telecommunication Engineering from the University of Pune, Pune, India in 2009. He is currently working towards the Ph.D. degree from Department of Electronics Engineering, Indian School of Mines, Dhanbad, India. His current research interests include optimization of nanoscaled circuits.

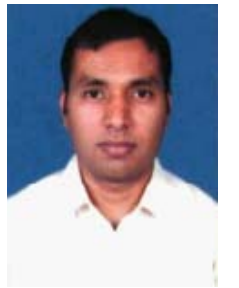

Ashok Kumar was born in Lucknow, India, in 1987. $\mathrm{He}$ received the B.Tech degree from Uttar Pradesh Technical University, India, in 2009, and M.Tech degree from Indian School of Mines, Dhanbad, India, in 2012. $\mathrm{He}$ is currently working as an Assistant Professor in the Department of Electronics and Telecommunication Engineering, Dr. D.Y. Patil School of Engineering, Pune, India. His current research interests include nanoscale MOSFETs and nanoscaled circuits.

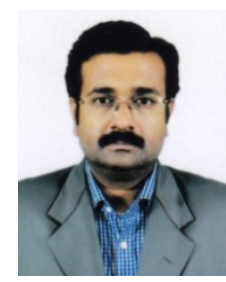

Subindu Kumar was born in Kolkata, India, in 1976. He received the B. Sc degree in Physics (Hons.) from University of Calcutta, India, in 1997, the M. Sc (Electronics) degree from Guru Ghasidas University (GGU), Bilaspur (M.P), India, in 1999 and M. Tech degree in Radio Physics and Electronics from University of Calcutta in 2001. He then joined Siliguri Institute of Technology (SIT), Siliguri (Dist: Darjeeling), India, as a faculty member. From 2004 he started working towards his Ph. D degree in Solid State Electronics (III-V Semiconductor Nanostructures) from the Institute of Radio Physics and Electronics, University of Calcutta. He completed his Ph. D in 2009. He served SIT from February 2001 to July 2009 in various capacities. He joined Indian School of Mines (ISM) Dhanbad on 21/07/2009 and is currently serving as Assistant Professor in the Dept. of Electronics Engg, ISM.

Dr. Kumar is the recipient of the University Gold Medal from GGU. He is a member of IEEE EDS, Photonics Society and a life member of Semiconductor Society of India (SSI). He has published more than 35 papers in peer reviewed international journals, national and international conferences. His research field includes semiconductor nanostructures, VLSI circuits and systems. 